Chirurg 2019 · 90 (Suppl 2):S53 https://doi.org/10.1007/s00104-019-0833-4 Online publiziert: 13. Februar 2019

(c) Springer Medizin Verlag GmbH, ein Teil von Springer Nature 2019

\section{Originalpublikation}

Daniels L, Ünlü Ç, de Korte N et al (2017) Randomized clinical trial of observational versus antibiotic treatment for a first episode of CT-proven uncomplicated acute diverticulitis. Br J Surg 104:52-61

Hintergrund. In den letzten Jahren wurde die Notwendigkeit einer Antibiotikatherapie bei der akuten unkomplizierten Divertikulitis wiederholt infrage gestellt. Mehrere Studien erbrachten Hinweise darauf, dass die alleinige Beobachtung eine sichere Alternative zur Antibiotikatherapie darstellen könnte.

Methoden. An 22 niederländischen Zentren wurden Patienten mit der ersten Episode einer CT-gesicherten akuten, unkomplizierten (modifizierte Hinchey-Klassifikation Stadien Ia-b), linksseitigen Divertikulitis randomisiert einer Beobachtungs- oder einer Behandlungsgruppe mit antibiotischer Therapie (Amoxicillin/Clavulansäure über $10 \mathrm{Ta}$ ge) zugeteilt. Primärer Endpunkt der Studie war die Zeit bis zur Genesung, definiert als die Kombination aus Entlassung aus dem Krankenhaus, normale Ernährung, Temperatur unter $38{ }^{\circ} \mathrm{C}$, geringer Schmerz-Score (VAS < 4/10) und Wiederaufnahme der beruflichen Tätigkeit.

Ergebnisse. Insgesamt 528 Patienten wurden in die Studie eingeschlossen.

Dieser Beitrag wurde erstpubliziert in Der Chirurg (2017) 88:344-344. https://doi.org/10. 1007/s00104-017-0417-0

M. Schrempf $\cdot$ M. Anthuber

Klinik für Allgemein-, Viszeral-, Transplantationschirurgie, Klinikum Augsburg, Augsburg, Deutschland

\title{
Ist eine Antibiotikatherapie beim ersten Schub einer unkomplizierten Divertikulitis erforderlich?
}

Zwischen der Beobachtungs- und Antibiotikagruppe war kein signifikanter Unterschied der medianen Zeit bis zur Genesung nachweisbar (14 Tage vs. 12 Tage; $p=0,15)$. Nach der initialen Diagnostik in der Notaufnahme wurden $13 \%$ der Patienten in der Beobachtungsgruppe und $0,4 \%$ in der Antibiotikagruppe ambulant behandelt $(p=0,006)$. Beobachtungs- und Antibiotikagruppe unterschieden sich nicht hinsichtlich der Wiederaufnahmerate $(17,6 \%$ vs. $12,0 \%$; $p=0,15)$, der Häufigkeit einer komplizierten Divertikulitis im Verlauf (3,8\% vs. $2,8 \%$; $p=0,38)$ und eines Rezidivs einer akuten Divertikulitis (3,4 \% vs. 3,0 \%; $P=0,49$ ). In der Beobachtungsgruppe erhielten 3,8\%, in der Antibiotikagruppe $2,3 \%$ der Patienten innerhalb von $6 \mathrm{Mo}-$ naten eine Sigmaresektion $(p=0,32)$. Antibiotikaassoziierte Nebenwirkungen traten bei $8,3 \%$ der Patienten in der Antibiotikagruppe und bei $0,4 \%$ in der Beobachtungsgruppe auf $(p=0,006)$.

Diskussion und Fazit. Diese Arbeit zeigt, dass die alleinige Beobachtung bei der ersten Episode einer akuten unkomplizierten Divertikulitis der bisher standardmäßigen, relativ intensiven antibiotischen Behandlung in den Niederlanden nicht unterlegen ist. Gestützt werden die Daten durch zwei retrospektive und eine randomisierte Studie [1]. Sofern engmaschige klinische Kontrollen gewährleistet sind, kann deshalb bei der unkomplizierten akuten Divertikulitis auf eine antibiotische Therapie verzichtet werden. In der vorliegenden Studie wurde auch das Stadium Hinchey Ib eingeschlossen, welches nach der Klas- sifikation der Divertikelkrankheit bereits einer komplizierten Divertikulitis entspricht. In diesem Stadium muss jedoch aufgrund mangelnder Daten vom Verzicht auf eine antibiotische Therapie noch abgeraten werden. Außerdem ist unklar, ob die Ergebnisse uneingeschränkt auf Patienten übertragbar sind, die nur eine sonographische Diagnostik erhalten. Zwar besitzt die Sonographie durch den erfahrenen Untersucher bei der Diagnosestellung der Divertikulitis eine vergleichbare Sensitivität wie die CT, allerdings ist die CT der Sonographie im Nachweis und in der Beurteilung von Komplikationen wie Abszessen und gedeckten Perforationen noch überlegen $[2,3]$.

\section{Korrespondenzadresse}

Prof. Dr. M. Anthuber

Klinik für Allgemein-, Viszeral-, Transplantationschirurgie, Klinikum Augsburg Stenglinstr. 2, 86156 Augsburg, Deutschland matthias.anthuber@klinikum-augsburg.de

Interessenkonflikt. M. Schrempf und M. Anthuber geben an, dass kein Interessenkonflikt besteht.

\section{Literatur}

1. Chabok A, Påhlman L, Hjern F et al (2012) Randomized clinical trial of antibiotics in acute uncomplicated diverticulitis. Br J Surg 99:532-539

2. Nielsen K, Richir MC, Stolk TT et al (2014) The limited role of ultrasound in the diagnostic process of colonic diverticulitis. World JSurg 38:1814-1818

3. Ripollés T, Agramunt $M$, Martínez $M J$ et al (2003) The role of ultrasound in the diagnosis, management and evolutive prognosis of acute left-sided colonic diverticulitis: a review of 208 patients. Eur Radiol 13:2587-2595 